\title{
Dificultades y proyecciones en la relación entre salud y espiritualidad
}

\author{
LEONARDO AgOSTINI FERNANDES \\ Pontificia Universidad Católica de Rio de Janeiro (Brasil) \\ laf2007@PUC-Rio.br
}

\begin{abstract}
Resumen
El presente artículo desea reflexionar, por un lado, sobre la necesidad de una formación integral de los agentes sanitarios, que contemple la dimensión espiritual; y, por otro lado, sobre la necesidad de una mejor preparación de los agentes religiosos que trabajan en hospitales y centros de salud, para que en sus intereses y preocupaciones por los enfermos, se eviten las dicotomías en nombre de la fe o en nombre de la razón. La promoción del diálogo entre razón y fe reduce la distancia entre las ciencias y evita el divorcio entre la praxis de la fe y la praxis de la ciencia, revelando que, a pesar de ser distintas por sus grados de certeza, pueden estar próximas por el objeto: la búsqueda de la verdad que libera al ser humano de todas las formas de injusticia y opresión, generadoras de malestar y enfermedades sicosomáticas.
\end{abstract}

Palabras claves: Ciencia, espiritualidad, fe, salud, verdad.

\section{Difficulties and projections on the relationship between health and spirituality}

\begin{abstract}
This paper intends to reflect, on one hand, the need for an integral education of healthcare agents, that addresses the spiritual dimension; and, on the other hand, the need for a better preparation of religious agents that work in hospitals and health centers, so that, in the interest and concern for sick people, dichotomies in the name of faith or in the name of reason are prevented. The promotion of dialogue between reason and faith shortens the distance between sciences and avoids a divorce between praxis of faith and praxis of science, showing that, despite the different degrees of certainty, may be close by the object: the search for the truth that liberates human beings of all forms of injustice and oppression, disease generators and psychosomatic illnesses.
\end{abstract}

Keywords: Science, Spirituality, Faith, Health, Truth.

Sacerdote de la Arquidiócesis de San Sebastián de Rio de Janeiro, Brasil. Doctor en teología bíblica por la Pontificia Universidad Gregoriana de Roma. Director y profesor de teología bíblica en el Departamento de teología de PUC-Rio y profesor de teología bíblica en el Instituto Superior de Teología de la Arquidiócesis de Rio de Janeiro. Entre sus publicaciones más recientes están: O Anúncio do Dia do Senhor. Significado profético e sentido teológico de Joel 2,1-11 (2014); "Por que morreremos na tua presença?: uma análise de Gn 47,13-26” (2014); y Evangelização e Família. Subsidio bíblico, teológico e pastoral (2015). 


\section{Introducción}

Empiezo con dos cuestiones: ¿Este tema ${ }^{1}$ representa una progresión, regresión o una intranquilidad meramente académica? No es muy común que la reflexión sobre la salud sea acompañada por el tema de la espiritualidad. ¿Entonces, en qué sentido el ejercicio de la espiritualidad debe insertarse en los debates y foros sobre la salud, ya sea ella tratada en el espacio público o privado?

Hace mucho tiempo que la salud se convirtió en el principal tema de debates y foros, en diferentes niveles, expresando la petición constante de personas en nuestra sociedad, tanto de los pacientes, que reclaman por un servicio mejor, como de los profesionales de la salud, que con razón, piden condiciones más dignas de trabajo. En muchos casos, los que deberían cuidar están dando claros señales de que necesitan recibir cuidados. En el fondo, el reclamo es por un sistema de salud más humano y menos dominado por intereses políticos y espurios, sin mencionar que esto es un clamor por la liberación de un sistema neocolonialista, a menudo cubierto bajo una apariencia democrática.

Tengo la intención de presentar mi reflexión sobre estas cuestiones, articulando el tema en tres puntos que siguen: 1) Dificultades en la relación entre salud y espiritualidad; 2) Proyecciones en la relación entre salud y espiritualidad; 3) Relación entre salud y espiritualidad más allá de las dificultades y proyecciones.

Al final de mi exposición, espero llegar no con respuestas acabadas, pero sí con propuestas que todavía puedan continuar alimentando la reflexión, sea personal o, como me gustaría, la reflexión de modo interdisciplinario e interinstitucional, porque creo que el ser humano, objeto de nuestros debates, no debería definirse solamente como Homo sapiens, capaz de adaptarse al medio para sobrevivir, mas como Homo collectivus, en cuanto capaz de juntar a los diferentes y de unir valores, superando obstáculos y creando puentes que relativicen las supuestas incompatibilidades entre los diferentes saberes.

\section{Dificultades en la relación entre salud y espiritualidad}

Este punto no es nuevo en la historia de la humanidad; dividida desde los albores del surgimiento y ascensión del conocimiento, entre los

\footnotetext{
1 Esta reflexión fue presentada en el II Simposio sobre salud y espiritualidad promovido por las instituciones: La Universidad del Rio de Janeiro (UNIRIO) y el Instituto Nacional de Traumatología y Ortopedia (INTO) de Rio de Janeiro en el día 24 de octubre de 2014.
} 
más cultos y los menos cultos, entre los más críticos y los menos críticos, para no decir, entre los que se consideran doctos y rotulan a los demás de ignorantes, mediocres o infantiles por creer en la existencia de un ser trascendente, que lo apellidaron «Dios». El problema fundamental fue, es y seguirá siendo, en medio de las luchas contra la fragmentación del conocimiento, o de la formación y no sólo de la información.

Sócrates, por ejemplo, ya reflexionaba, con sus discípulos, sobre la necesidad de la oferta de animales a Esculapio $^{2}$ para la obtención de la salud, de la cura de enfermedades o la eliminación de posibles males individuales o familiares, en particular de la muerte ${ }^{3}$. Sin embargo, en la percepción criteriosa del filósofo, había respeto por las creencias y, en ellas, no se veía sólo una obligación, sino el reconocimiento de que la religiosidad es una dimensión intrínseca del ser humano. Hoy, se evocaría la cuestión metodológica con que el ser humano llegaría a acceder a la realidad y, en ella, conseguir posicionarse críticamente.

En efecto, el cuestionamiento del filósofo era con respecto a la etiología de las enfermedades. Por ejemplo: los disturbios que escapaban al control o a la normalidad familiar y social eran atribuidos a la influencia de los demonios, de fuerzas espirituales o a causas naturales. Estos disturbios, más tarde, fueron correctamente clasificados, por la ciencia, como epilepsia, neurosis, bipolaridades, etc. Sobre esto, sin embargo, se impone un paréntesis: la cura para estos males todavía no está en las manos de la ciencia que, a lo máximo, interviene con un medicamento con base de neurotransmisores, asistida, en la mayoría de los casos, por la terapia y cercanía psicológica. A pesar de esto, no es raro ver a personas afectadas por dichas enfermedades llevadas por familiares o amigos a secciones con curanderos o exorcistas.

Sabemos que la historia humana es el entablado de la aparición de enfermedades, como de la lucha contra ellas. Una breve mirada en el curso de la medicina, desde Hipócrates (c. 410 A.C. $)^{4}$, hasta los días ac-

2 En la mitología griega, era el dios de la medicina, hijo de Apolo y de la ninfa Coronis, que aparece citado en la Ilíada de Homero (siglo VIII A.C.), como un médico calificado, pero no aún divinizado (Homero, 2009)

3 Antes de su muerte, Sócrates habría dicho a su discípulo Critón: «Somos deudores de un gallo a Esculapio, no te olvides de pagar esa deuda» (Esculapio, 2015). ¿Esto sería verdad? ¿Ironía o fe? Creo que estamos lejos de alcanzar una respuesta.

4 Para Hipócrates, la salud es la proporción exacta entre los humores y las fuerzas en su justa medida y en su equilibrio ordenado. Esta definición, por extensión, debería ser comprendida como equilibrio del ser humano con el medio ambiente y con la sociedad. Así se podría clasificar como enfermedad la falta de equilibrio en uno de estos aspectos. Entonces, tener cuidado de la salud sería visto como un proceso mediante el cual se ayuda a una persona a tener su equilibrio restaurado en sí misma, con la socie- 
tuales, revela que siempre hubo la búsqueda por la sanación de los males $\mathrm{o}$, al menos, el alivio del dolor y del sufrimiento, para que cada persona lograse ser un protagonista de su acto de morir con dignidad; lamentablemente, en nuestros días, eso representa más un deseo de índole retórica que un factor constatado en la práctica general.

La tarea es una lucha en favor de la vida y contra todo lo que sería la causa de la muerte. Así, repugna cualquier tipo de intervención que conduzca a la muerte de un ser, sea al principio o al final de su vida (Fernandes, 2014: 141-158). En dicha dinámica, está el interés y el compromiso por la origen del mal, es decir, por la etiología de las molestias y las enfermedades, siempre buscando la salud, o mejor, la salvación, término que deriva del griego soter y que llegó a las lenguas neolatinas a través del Latín salus, salutis. Al menos etimológicamente, hay una estrecha relación entre salud y salvación ${ }^{5}$, un término comúnmente usado en los ambientes religiosos. No es infrecuente, sin embargo, escuchar de una persona agradecida: «Doctor(a), usted me salvó la vida!».

Todavía, desde el periodo hipocrático hasta el positivista, no existía, en la mirada sobre la realidad, una separación entre el mundo físico y el mundo espiritual. En la verdad, ellos aún no eran concebidos como «dos mundos», pero sí privilegiaban la realidad como una. De tal manera que, tratar de lo corporal era tratar de lo espiritual y tratar de lo espiritual era tratar de lo corporal. En muchas culturas, el curandero de un grupo también era el sacerdote de la comunidad.

En varios países, particularmente en América Latina, no es difícil de percibir que las distancias culturales son, en muchos casos, alarmantes; razón por la cual las dificultades surgen, en la mayoría de los casos, por la falta de formación y por el progresivo alejamiento generado entre fe y ciencia, resultante de la falta de visión unificada y psicosomática del ser humano. La primera se considera ingenua o «inteligencia de inferiores», mientras que la segunda es tomada por seria y criteriosa o «inteligencia de superiores». En este sentido, una gran dificultad a ser superada es disminuir la distancia entre lo que sabe el agente sanitario y lo que sabe el paciente, que, en gran parte, no pide respuestas teóricas, sino si llegará o no a la cura.

En este contexto, la percepción y la comprensión de la realidad del agente sanitario y de cada paciente pasarán, necesariamente, por el ins-

dad y con el ambiente en que vive. En teoría, entonces, salud y enfermedad no son conceptos abstractos, porque sólo existen en los seres que están vivos.

5 Por salvación se entiende la liberación de toda clase de mal que amenace el ser humano tanto en su cuerpo como en su alma. En este sentido, salvación y liberación son términos que pueden ser considerados no sólo como sinónimos, pero capaces de traducir, inclusive, situaciones no religiosas. 
trumental de la inteligencia de cada uno y por la trayectoria del pensamiento que surgió y se desenvolvió en el mundo occidental hasta llegar a un progreso decisivo: el cambio de una visión religiosa del mundo a una visión secular, que continúa su papel y ha influido mucho en las posiciones sociales (Tarnas, 2011: 343). Cada vez más, los gobiernos declaran que el Estado es laico, es decir, independiente de cualquier vínculo con una institución religiosa. Tal declaración, sin embargo, no obliga en conciencia, la prohibición de la práctica y de la asistencia religiosa a los pacientes que la soliciten, junto al servicio de salud, la ayuda espiritual y religiosa según la fe que ellos libremente profesan ${ }^{6}$.

En los últimos dos siglos, sin embargo, las corrientes de pensamiento racionalistas y fideístas intercambiaron numerosas y recíprocas censuras. El primer grupo, acusando el segundo de sólo apreciar el espíritu, disminuyendo el valor del cuerpo. El segundo grupo, acusando el primero de solamente cuidar de la materia, despreciando el alma. Por eso, en muchos medios académicos pasó a predominar el escepticismo y hasta la total aversión a la dimensión religiosa y, en muchas corrientes religiosas, la aversión por los médicos y por los agentes sanitarios, considerándolos como ateos o no creyentes.

En parte, esa aversión fideísta deriva de la "religión de los escépticos", que apareció en Francia en el siglo XIX, con una postura cientificista y positivista que se niega a «admitir, como válidas, las formas de conocimiento diversas de las propias Ciencias positivas» (Francisco, 2013: 242). En contraste, el cerramiento recrudecido de racionalistas y de seguimientos académicos al conocimiento religioso no está desprovisto de razón y fundamentos. La religión cristiana, católica o protestante, cargan con una historia de diversas formas de condenación que fueron impuestas a los hombres de ciencia, por el simples hecho de tener una hermenéutica de la realidad diferente del poder religioso que tomaba las afirmaciones bíblicas como verdades científicas incuestionables ${ }^{7}$. A esto

6 En el Título II - Sobre los Derechos y Garantías Fundamentales; Capítulo I Sobre los Derechos e Deberes Individuales y Colectivos, Art. $5^{\circ}$ párrafo VI de la Constitución del Brasil se dice: «es inviolable libertad de conciencia y creencia, visto que se garantiza el libre ejercicio de los cultos religiosos y está garantizada, en forma de ley, la protección de los lugares de culto y sus liturgias». Y en el párrafo VII se dice: «a nadie se privará los derechos por motivo de creencia religiosa o convicción filosófica o política, a menos que el las invoque para evitarse de la obligación legal a todos impuesta y negarse a cumplir la ayuda alternativa, fijada en la ley» (Constitução da República Federativa do Brasil, 2015: art 5).

El tema de las verdades bíblicas asumió una nueva perspectiva con la Constitución Dogmática Dei Verbum, del Concilio Vaticano II, 3-4. La Pontificia Comisión Bíblica publicó recientemente, un nuevo documento, que, en particular, trata de la inspiración y la verdad de la Biblia, afirmando que: «No hay duda de que la Verdad, que se encuentra 
hay que agregar un gran pesar, cuando un paciente, diagnosticada su enfermedad, interrumpe o incluso no empieza un tratamiento, motivado por los fundamentalismos religiosos que prometen curas en nombre de Dios o prohíben los tratamientos porque necesitan de una transfusión de sangre.

Todavía, es necesario tener en cuenta la sensibilidad del Papa Francisco que dice:

La Iglesia no pretende detener el admirable progreso de las ciencias. Al contrario, se alegra e incluso disfruta reconociendo el enorme potencial que Dios ha dado a la mente humana. Cuando el desarrollo de las ciencias, manteniéndose con rigor académico en el campo de su objeto específico, vuelve evidente una determinada conclusión que la razón no puede negar, la fe no la contradice (...). Pero, en ocasiones, algunos científicos van más allá del objeto formal de su disciplina y se extralimitan con afirmaciones o conclusiones que exceden el campo de la propia ciencia. En ese caso, no es la razón lo que se propone, sino una determinada ideología que cierra el camino a un diálogo auténtico, pacífico y fructífero (Francisco, 2013: 243).

Este aislamiento podría encontrarse en la definición de salud presente en la Constitución de la Organización Mundial de la salud (OMS), aprobada en 22 de julio de 1946, en Nueva York, cuando definió la salud como «Un estado de completo bienestar físico, mental y social y no solo la ausencia de enfermedades». Nótese que el factor espiritualidad no fue contemplado. Esto solamente fue incorporado, pero no sin fuertes críticas y polémicas, en 2003 por la $\mathrm{OMS}^{8}$, que redefinió el término salud como «un proceso armonioso de bienestar físico, psíquico, social y espiritual», y no exclusivamente la ausencia de enfermedades?

Por esta nueva definición, podemos individualizar las posibles causas de las dolencias y enfermedades: a) carencia de bienestar físico; b) caren-

en el centro de la revelación y, consecuentemente, en el centro de la Biblia como un instrumento para la transmisión de la revelación (cf. Dei Verbum, nn. 7-10), se refiere a Dios y a la salvación del ser humano. Y no hay duda que la plenitud de tal verdad está manifestada por y en Jesús Cristo» (Pontificia Comissão Bíblica, 2014: 65). He tratado recientemente sobre el tema de la verdad (Fernandes \& Amado, 2014: 97-116).

8 La inclusión del elemento espiritual, en la redefinición del término salud, abrió espacio para que se realicen pesquisas en cuanto al factor de impacto de creencias, religiones y espiritualidad en la vida de los pacientes y agentes sanitarios (Volcan, Sousa, Mari \& Horta, 2003; Fleck, Borges, Bolognesi \& Rocha, 2003; Sanchez \& Nappo, 2008; Panzini, Maganha, Rocha, Bandeira \& Fleck, 2011).

$9 \quad$ Esta definición fue retomada por el CELAM, a la cual incluyó: «proceso que capacita el ser humano a cumplir la misión que Dios le ha destinado, según la etapa y las condiciones de vida en la cual se encuentra» (CELAM, 2012: 6-7). 
cia de bienestar psíquico $^{10}$; c) carencia de bienestar social; d) carencia de bienestar espiritual. En otras palabras, podemos decir que la salud resulta del equilibrio corporal, psíquico y espiritual, experimentado y amparado en los distintos niveles sociales: familia, trabajo, escuela, diversión, círculos de amigos, iglesia, lugares de celebración, etc. A partir de esta nueva definición, a los espacios de tratamiento de enfermedades y de recuperación de la salud, deberían integrarse también la atención espiritual, desde la perspectiva de la atención integral.

Al final, incluso en este punto, cabe mencionar que la espiritualidad no es un concepto unívoco y frecuentemente es confundido con la opción o práctica religiosa del enfermo o del agente sanitario. En la tradición cristiana, la espiritualidad también no es unívoca y se desarrolla en un abanico de posibilidades unificadas, sin embargo, con un elemento común: la noción de que la espiritualidad es la acción del Espíritu Santo en la vida de los bautizados ${ }^{11}$. En este sentido, la espiritualidad es vida según el Espíritu Santo, para que cada uno de los bautizados viva en y del misterio de Jesucristo. Esta inmersión en Jesucristo acontece como un camino de perfección personal por los dones y por los carismas colocados al servicio de los hermanos en la fe y, entre ellos, está el don para obtener la cura o para hacer milagros. El apóstol Pablo dio sistematización a estos conceptos en 1Cor 12,4-11.

\section{Proyecciones en la relación entre salud y espiritualidad}

En los últimos años, las enfermedades clásicas se redujeron considerablemente $y$, en muchos lugares, pasaron a ser consideradas erradicadas; pero, en contrapartida, otras surgían y han puesto nuevas preguntas e inquietudes a las organizaciones sanitarias. Únase a esto que, por un lado, la media de vida ha aumentado; y por otro lado, también aumentarán las probabilidades de las enfermedades derivadas de edad anciana, como el Alzheimer y los varios tipos de cáncer. En muchos casos, prolongar la vida pasó a significar el prolongar la posibilidad del dolor y el sufrimiento

\footnotetext{
10 Según la psicología, la salud tiene que ver con la condición plena de eficiencia funcional que, en el ser humano, comprende también funciones lógicas, afectivas, relacionales en contestos interpersonales y sociales. En este sentido, la salud está mucho más allá de la ausencia de enfermedad y, por tanto, no puede ser reducida a la mera categoría médica (Galimberti, 2006: 381).

11 «La espiritualidad dice respecto al cultivo de una dimensión fundamental, que se ocupa de la interioridad del ser humano, que envuelve la 'expansión de la vitalidad' y de la calidad de vida. Un camino que rescata una concepción más fecunda del ser humano, en particular su dimensión de profundidad, que escapa a los parámetros transmitidos por la cultura dominante» (Teixeira, 2014: 151).
} 
para millones de personas, independientemente de las condiciones de buena o mala salud pública. Nuestra especie humana no sólo vive más tiempo que las otras, por su característica biológica, sino también porque es capaz de prolongar su existencia gracias a su inteligencia. Ante esto, preguntase: ¿Es posible llegar a la tercera edad sin enfermedades? Ya sabemos que la ausencia de enfermedades no es sinónimo de salud.

La famosa expresión, mens sana in corpore sano, del poeta romano Juvenal (Sátira X) (2) $^{12}$ permanece utilizada por muchos, pero con sentidos que no siempre son coherentes con el núcleo de su formulación. Juvenal entendió que la oración no debía ser utilizada con fines espurios, sino para pedir a los dioses lo que realmente importaba; en este caso una mente sana en cuerpo sano para realizar los servicios que enaltecen la vida. En el fondo, por detrás de esa expresión estaba una finalidad: la mente sana desea estar en un cuerpo sano y un cuerpo sano quiere ser impulsado por una mente sana. Nótese claramente la profunda relación entre lo físico y lo psíquico, o entre el corpóreo y lo espiritual.

La vida y la muerte, junto a la salud y a la enfermedad, no son temas que solamente interesan y fomentan los debates académicos; también son realidades que se encuentran envueltas por una esfera misteriosa. Por misterio, entiendo un fenómeno ante lo cual la ciencia no tiene una explicación y para la cual no consigue formular un parecer según las verdades que ya ha alcanzado y según la certeza de que profesa, casi como si fueran dogmas. Para decirlo en otras palabras, el misterio es algo que atormenta a los que, siendo devotos exclusivamente al culto de la ciencia, viviendo una especie de 'vocación religiosa', no admiten la falta de respuestas para determinadas situaciones que deberían estar bajo el control de un aparato tecnológico avanzado y disponible en el mercado.

Es en este contexto, deseo colocar dos preguntas. ¿Por qué los fármacos de última generación, los médicos ultra bien formados ${ }^{13}$ y hospitales altamente sofisticados no consiguen restituir la salud para tantos pacientes que, a su vez, no responden a los tratamientos como se esperaba?

12 Disponible en http://www.nimispauci.com/index.php.

13 La medicina actual está inmersa en una tela de especialidades necesarias, pero envueltas en paradojas. Hay, en un cierto sentido, la nostalgia por antiguos médicos generales, considerados más bien preparados por su formación más humana y social; por sus capacidades de ver y de cuidar de la persona como un todo. Si, por un lado, se han multiplicado las clínicas, los médicos clínicos generales son cada vez más escasos debido a factores pedagógicos y particularmente financieros. Las universidades son a menudo muy técnicas, haciendo prevalecer el valor de los equipamientos en detrimento de la semiología. En la práctica, la medicina diagnóstica, apoyada por la tecnología, alcanza más lucros que la medicina clínica o de intervención. Nótese el desfase entre lo que el paciente paga y lo que el profesional sanitario recibe, hechas sin duda las honorables excepciones. 
¿Qué acontece entre el diagnóstico de una enfermedad y su tratamiento que, al final de las cuentas, resulta ineficaz? ¿Por qué en el campo científico milita tanta dificultad para integrar, en el proceso de atención e cuidados del paciente, el importante papel de las religiones?

Estas cuestiones, cuando son llevadas en justa consideración, abren la mente y el corazón de los académicos para el misterio; una realidad que, aunque presente, escapa al control científico. Es en este momento que muchos pasan a admitir el milagro como una posibilidad (la comprensión actual de milagro no implica ir contra la naturaleza de las cosas o personas) donde las posibilidades humanas dejaron de existir o se han agotado. La ciencia misma, aunque no reconozca el milagro, reconoce que se encuentra frente a fenómenos que están más allá de sus posibilidades de comprensión ${ }^{14}$. Es verdad, también, que el hilo que separa la cura por la fe de la cura por la ciencia es muy tenue y no hay escasez de especuladores y charlatanes que no tienen como objetivo el bienestar de los enfermos, sino utilizan su debilidad psíquica para infundir falsas esperanzas.

Por otro lado, si tomamos las tradiciones religiosas del judaísmo, del cristianismo y del Islam, y las comparamos, percibiremos que hasta el final de la Edad Media, no hubo gran separación entre el mundo físico y lo espiritual, entre la salud corporal y espiritual, hubo más bien interacción. Me gustaría tomar el judaísmo como un ejemplo de la difusión de la higiene en el mundo occidental; del cristianismo como un ejemplo de cuidado con los enfermos a través de las distintas órdenes religiosas que se convirtieran en renombrados hospitales y del Islam como un ejemplo de desarrollo de la medicina. El avance del Islam, particularmente en esta área, fue tan notable que, ya en el siglo décimo, existían manuales de anatomía y una fructífera investigación de las relaciones entre enfermedades y medicamentos catalogados en forma de manuales. El mundo occidental es, en gran parte, deudor de la investigación realizada por los sabios y religiosos musulmanes.

Así como una atención médica no sería eficaz si no tomara en cuenta los gemidos espirituales de un enfermo, de igual modo, una atención espiritual no sería auténtica si no tomara en cuenta los gemidos corpóreos de un enfermo. Es necesario reconocer una verdad elemental: el enfermo que gime, sea de forma física o espiritual, es una persona que tiene no sólo el derecho al tratamiento corporal más adecuado, sino también tiene el derecho al acompañamiento espiritual. Asegurar un derecho

\footnotetext{
14 Las universidades empiezan asumir un importante protagonismo en la aproximación y promoción del diálogo entre los diversos campos de las ciencias. Cito tres ejemplos: Soares \& Passos, 2008; Da Cruz, 2011; García Rubio \& Amado, 2012.
} 
y no permitir el otro, sería simplemente una manera de mantener una visión reducida del ser humano, volviendo a la dicotomía entre el corporal y lo espiritual.

En general, la recepción o la acogida de la asistencia religiosa es una decisión del enfermo y no de aquel que está como gestor del servicio sanitario, sea público o privado. Eso es lo que dice, en el caso de Brasil, el Art. $5^{\circ}$ de la Carta de los derechos de los Usuarios de salud (2011) «Toda persona debe tener sus valores, cultura y derechos respetados en la relación con los servicios de salud, asegurando para él (...) la recepción o el rechazo a la asistencia religiosa, psicológica y social».

Las investigaciones muestran el alto índice de sanación en los pacientes que recibieron, de los dos lados, una atención más humana y altruista ${ }^{15}$. Haciendo una comparación y análisis de datos, los hospitales dirigidos por denominaciones religiosas lograron mejores resultados en comparación con centros clínicos y hospitales dirigidos solamente de forma secular y que no integran el apoyo espiritual de sus pacientes. Y aún cuando el paciente terminó falleciendo, la preparación para la muerte aconteció de manera más humana y digna, tanto en la vida del paciente como de los familiares. La fe y la espiritualidad, cultivadas juntas con la certeza de que los equipos sanitarios trabajaron con atención y cuidado, aumentarán el confort y el consuelo en la hora de la muerte.

\section{Relación entre la salud y la espiritualidad más allá de las dificul- tades y proyecciones}

No es necesario ser un perito para darse cuenta que, desde la parte de las grandes conquistas tecnológicas en el área de la salud, existe una gran crisis generalizada en las instituciones que se ocupan de la salud, sean públicas o privadas. Junto a todos los logros tecnológicos, existe igualmente el progreso, yo diría, no tanto de las enfermedades, sino del número de enfermos. Algo que se explica sin grandes dificultades por el crecimiento demográfico y por el aumento del tiempo de la vida. Con lamento, se percibe que acontece una mayor atención a la enfermedad que al enfermo.

¿Sería posible decir que, en el pasado, las personas eran más saludables y menos propensas a las enfermedades, a pesar de la precariedad

15 «La idea de que ciencia y espiritualidad son áreas antagónicas son parte del pasado. Investigaciones realizadas en países como Brasil, Canadá y Estados Unidos buscan demostrar cómo las experiencias de índole espiritual ayudan a mejorar la calidad de vida de las personas. Esta tendencia se está afirmando hace unos años y adquiere mayor protagonismo con el aumento de los estudios sobre el asunto» (Teixeira, Müller \& Da Silva, 2004: 8). 
sanitaria de los muchos sitios? ¿Es posible admitir que ciertas enfermedades son causadas por la ruptura del ser humano con la totalidad de su existencia y que la cura puede acontecer, haciendo el camino de retorno a esa totalidad? ¿En qué sentido la falta de felicidad o la pérdida del sentido de la vida son generadores de enfermedades?

Para dirimir estas cuestiones, no creo que bastaría una simple estadística, pero sería necesario un estudio que, además de proponer datos estadísticos, lograse clasificar y mapear la aparición y evolución de las enfermedades en el mundo y para hacer el link con los factores generadores de felicidad y de sentido de la vida, como en el caso de la fe y la espiritualidad.

Por cierto, el estudio de la aparición y evolución de las enfermedades ya existe y la OMS tiene los datos y, de vez en cuando, presenta el desarrollo o el retroceso de enfermedades en los diferentes países. Estos son inclusos bien clasificados en la medida que disfrutan de políticas de salud capaces de erradicar las enfermedades. Sin embargo, los factores, felicidad y sentido de la vida, en gran parte, cuando aparecen en las estadísticas, figuran como satisfacción en la asistencia, en el tratamiento o como un sinónimo de ausencia de enfermedades.

Estos estudios tienen en cuenta que enfermedades desarrolladas en las zonas rurales no tienen la misma incidencia de las que existen en las zonas urbanas y viceversa. El curso de las enfermedades depende de la vulnerabilidad, de la incidencia y del volumen de la movilidad humana. En este sentido, las enfermedades contraídas y de mayor proliferación son relativamente proporcionales al flujo humano de los lugares donde están presentes para los lugares donde todavía no habían sido detectadas. Es suficiente el recuerdo de la preocupación internacional, en las últimas décadas, con el virus del SIDA y el Ébola, diagnosticado por primera vez en 1976, en surtos simultáneos en el Sudán y en el Congo, a los márgenes del Río Ébola.

Mientras que la filosofía antigua parece haberse ocupado de la salud del alma y de la salud del cuerpo, en la actualidad, gracias al desarrollo de la medicina más orientada al ser humano de forma integral, una nueva cultura de la corporalidad parece presentarse, dando espacio para que se trate no sólo del físico, sino también, en forma integrada, de lo psicológico, lo moral y lo espiritual.

Este espacio permite a la persona percibir que tener o cultivar una espiritualidad o una creencia religiosa mejora la salud mental, pues proporciona al ser humano una apertura para relacionarse con el mundo teniendo buenas intenciones y propósitos, enriqueciéndolos de significado y de esperanza, promoviendo su bienestar; también mejora la salud en el nivel social, pues el encuentro con el prójimo de forma altruista favo- 
rece la mayor estabilidad y equilibrio psicosomático. Con eso, comportamientos más saludables son priorizados y desarrollados, el fumar, el alcohol, las drogas y las prácticas sexuales peligrosas o por mera compulsión.

La medicina, desde tiempos remotos, siempre ha tenido en cuenta el impacto de la espiritualidad sobre el factor salud, pero los estudios científicos sobre esta incidencia son relativamente recientes. Así, la cuestión de la salud y la espiritualidad se abre para contemplar el horizonte antropológico de forma más amplia, favoreciendo la relación entre el agente sanitario y el enfermo; se pone en evidencia el nexo entre el ser humano, la enfermedad y el proceso que envuelve todo y cualquier tipo de tratamiento, visando la cura o el alivio del dolor y del sufrimiento. Con esto, se busca humanizar tanto el médico como el paciente, a través de una relación que reclama por una educación moral y ética, tanto del conocimiento como del comportamiento.

Es en esta apertura que la promoción de la espiritualidad puede traer beneficios no sólo a los enfermos, sino también para los agentes que trabajan en la salud. La espiritualidad puede contribuir grandemente a la humanización de los centros que promueven la salud y el bienestar de las personas. En la medida en que se favorece la relación con la vida plena, se permite que los espacios de tránsito humano sean impregnados de la fuerza que favorece una profunda y consciente apertura a nuevas experiencias que generen conocimientos.

En la medida en que la espiritualidad pasa a ser vista como un valor que agrega, la solidaridad, el respeto y la compasión manifiestan, de manera más intensa, la grandeza de la dignidad del ser humano frente al modo en frío como el cientificismo racionalista pasó a dictar las reglas sobre cómo los enfermos debían ser tratados. Además de las dificultades y de las proyecciones, estamos contemplando un nuevo horizonte, en que el diálogo entre las ciencias acontece gracias a la apertura y el interés por la prioridad que debe darse al ser humano. La promoción del diálogo entre razón y fe reduce la distancia entre las ciencias y evita el divorcio entre la praxis de la fe y la praxis de la ciencia, mostrando que, a pesar de los distintos grados de certeza, pueden ser próximas por el objeto: la búsqueda de la verdad que libera al ser humano de todas las formas de injusticia y opresión que son generadoras de enfermedades. 


\section{Consideraciones finales}

Creo que promover la «religación de los saberes» ${ }^{16}$ es el gran desafío para los hombres y mujeres de buena voluntad del nuestro siglo. No sería insano pensar que la medicina podrá avanzar en la búsqueda de una solución para el problema que se impone entre la salud y la enfermedad, en la medida que ya no se considere sólo una ciencia natural aplicada, abriéndose también a lo que las ciencias humanas y sociales tienen que ofrecer.

Sería útil y oportuno un trabajo de revisión bibliográfica sobre lo que ya se ha hecho en América Latina sobre el tema de la salud y la espiritualidad ${ }^{17}$. Tal vez el resultado no sea muy alentador, pero ayudaría en el desarrollo de líneas de investigación capaces de mejorar la formación y la capacitación de operadores sanitarios, de los profesionales y de los voluntarios que prestan servicio religioso en clínicas y hospitales.

En contrapartida, la espiritualidad para que en el fruto sea expresión de una fe genuina, debe pasar por un proceso de purificación de todas las formas de fundamentalismos. Esto es, por cierto, un éxodo necesario de las formas religiosas que, en lugar de ayudar la liberación de la persona, se convirtieron en un instrumento de opresión. Muchos, por eso motivo, ya no quieren seguir una denominación religiosa y pasan a vivir una espiritualidad meramente intimista y sin vínculos. En el fondo, no es sólo la manifestación del ser humano que reacciona contra el vacío producido por sistemas antirreligiosos, sino también por la falta de coherencia de vida de los que dicen ser religiosos. En oposición a eso, sería una falta de

16 En el mundo actual fragmentado, hombres y mujeres, comprometidos con la formación humana integral, claman por la aparición de principios organizadores del conocimiento, visando la superación de las dicotomías generadas por la fragmentación del saber. Comparto la afirmación de Ardoino (2010: 558): "Querer reunir saberes no implica el desarrollo de una transdisciplinaridad homogeneizadora, pero lleva, aún así, a situarlos con precisión de unos en relación a los otros en función de sus alteridades históricas, antropológica y epistemológicas (sin, por eso, eliminar sus posibilidades de mutua alteración)».

17 La Campaña de la Fraternidad promovida por la Conferencia Nacional de los obispos del Brasil (CNBB) como acción cuaresmal concreta, ha trabajado, con profundidad, el tema Fraternidad y la Salud Pública en 2012. El Manual, intitulado Que la salud se extienda sobre la tierra (cf. Eclo 38,8), ofrece, en el Texto de Base, una reflexión muy rica y detallada sobre la realidad de la salud pública en Brasil. Siguiendo el método ver, juzgar y actuar, hace una lectura de la realidad y ofrece preciosas indicaciones a nivel personal, eclesial y social. De forma académica, surgió un núcleo de investigaciones en la Universidad Federal de Juiz de Fora en el programa de postgrado en salud. Para obtener más informaciones, véase: http://www.ufff.br/nupes/. 
fe y de espiritualidad provocar actitudes pesimistas, de desconfianza y de ideas fatalistas.

En nuestro proceso de hominización está un elemento diferenciador de las demás etapas evolutivas de los animales: el ser humano es el único capaz de obedecer a un raciocinio y de organizarse en función de las consecuencias que puede prever, visando mejorar sus condiciones de vida y de conservación. Esta lógica es un dado genético, inscrito en su $\mathrm{ADN}$, que viene desarrollando en cada etapa de su existencia superada y a ser superada.

No contradice la existencia de Dios la aceptación de que la historia tiene incontables potencialidades y capacidades aún no cuantificadas o catalogadas. Yo, personalmente, creo que: «La salud y su opuesto, la enfermedad, deben ser consideradas como fenotipos vinculados a la acción conjunta de una agresión (generalmente exterior) y de un desreglamento interno que los mecanismos de homeostasis no llegan ser suficientes para rectificar» (Ruffié, 2010: 250) ${ }^{18}$. En este sentido, la espiritualidad, sanamente cultivada, puede ser un mecanismo valioso, una reacción química capaz de desencadenar nuevos mecanismos homeostáticos que permitan el ser humano no ser solamente el protagonista de sus enfermedades, pero, principalmente, de sus procesos de sanación física y psicológica. En muchos casos, la oración, como la de los Salmos bíblicos, es un vehículo capaz de devolver la paz a los afligidos.

En la lucha diaria contra los sufrimientos y las enfermedades, no se deberían suprimir la fe y la espiritualidad. Poner una mirada sobre la relación entre salud y espiritualidad permite ver, con criterio, que para ser saludable el ser humano necesita integrar a lo físico y a lo mental, lo espiritual, mostrando lo que eso puede hacer en la vida de los agentes y pacientes ansiosos por salud. Los desafíos y las proyecciones en la relación entre salud y espiritualidad necesitan ser juzgados según el valor de la vida de la persona humana. Así, es igualmente necesario generar en el sistema de salud, la solidaridad, la fraternidad, la igualdad y el interés por el bienestar común.

$\mathrm{Si}$, por un lado, la previsión es una característica del Homo sapiens que lucha por su conservación; por otro lado, él no puede predecir todo, pero podría evitar para él mismo muchas cosas, inclusive de la mayoría de las enfermedades si empezase a ejercitar mejor su dimensión de Homo collectivus contra todas las formas de aislamiento. Vivir no es sólo un imperativo, sino una vocación, que se realiza, con competencia, en la medi-

18 Homeostasis es el proceso de regulación por medio del cual un organismo mantiene su equilibrio constante. Fue un término acuñado por el fisiólogo norteamericano Walter Cannon (1871-1945). 
da en que las relaciones establecidas proporcionan el equilibrio entre el saber científico y el espiritual, produciendo la salud integral del ser humano.

\section{REFERENCIAS}

-Ardoino, J. (2010). A Complexidade. En E. Morin (Coord.), A Religação dos Saberes. O desafio do século XXI (pp. 548-558). Rio de Janeiro: Bertrand Brasil.

-CELAM (2012). Guia para a Pastoral da Saúde na América Latina e no Caribe. São Paulo: Centro Universitário São Camilo.

-Conferência Nacional dos Bispos do Brasil (2012). Que a saúde se difunda sobre a terra (cf. Eclo 38,8). Brasília: Edição CNBB.

-Concilio Vaticano II (2000). Dei Verbum (Segunda edición). (L. Amigo \& R. Herrera, Trads.) Madrid: BAC.

-Constitução da República Federativa do Brasil (2015), Recuperado en http://www.senado.gov.br/atividade/const/con1988/CON1988_07.05.2 $015 /$ ind.asp

-Da Cruz, E. (2011). Teologia e Ciências Naturais. Teologia da Criação, Ciências e Tecnologia em diálogo. São Paulo: Paulinas.

-Esculapio (2015). Lessico. Recuperado en http://www.summagallicana.it/lessico/e/Esculapio\%20o\%20Asclepio.ht $\mathrm{m}$

-Fernandes, L. \& Amado, J. (2014). Evangelii Gaudium em Questão. Aspectos bíblicos, teológicos e pastorais. São Paulo: Paulinas.

-Fernandes, L. (2014). Como entendemos o Final da Vida. A morte e o morrer à luz da revelação bíblica e da teologia. Revista de Cultura Teológica, 22, 141158.

-Fleck, M., Borges, Z, Bolognesi, G. \& Rocha, N. (2003). Desenvolvimento do WHOQOL, módulo espiritualidade, religiosidade e crenças pessoais. Revista de Saúde Pública, 37(4), 446-455.

-Francisco (2013). Evangelii Gaudium. Brasília: Edição CNBB.

-Galimberti, U. (2006). Dizionario di Psicologia (Vol. 3). Roma: L'Espresso.

-García Rubio, A. \& Amado, J. (2012). Fé Cristã e pensamento evolucionista. Aproximaçoes teológico-pastorais a um tema desafiador. São Paulo: Paulinas.

-Homero (2009). Ilíada. (M.O. Mendes, Trad.) Versión para ebook http://www.ebooksbrasil.org/adobeebook/iliadap.pdf

-Juvenal, Décimo Junio. SATIRE X. (Traducción de Sueli Ratto). Versión francesa del original en latín. Disponible en http://www.nimispauci.com/index.php.

-Ministério de Sáude (2011) Carta dos direitos dos usuários da saúde (Tercera edición). Brasilia: Série E. Legislação de Saúde

-OMS (1946). Constitución de la Organización Mundial de la salud. Recuperado en http://apps.who.int/gb/bd/PDF/bd47/SP/constitucion-sp.pdf?ua=1 
-Panzini, R., Maganha, C., Rocha, N., Bandeira, D. \& Fleck, M. (2011). Validação brasileira do Instrumento de Qualidade de Vida/espiritualidade, religião e crenças pessoais. Revista de Saúde Pública, 45(1), 153-165.

-Pontificia Comissão Bíblica (2014). Inspiração e Verdade da Sagrada Escritura. A palavra que vem de Deus e fala de Deus para a salvação do mundo. São Paulo: Paulinas.

-Ruffié, J. (2010), Biologia humana e medicina de predição. En E. Morin (Coord.), A Religação dos Saberes. O desafio do século XXI (pp. 248-250). Rio de Janeiro: Bertrand Brasil.

-Sanchez, Z. van der M. \& Nappo, S. (2008). Intervenção religiosa na recuperação de dependentes de drogas. Revista de Saúde Pública, 42(2), 265-272.

-Soares, A. \& Passos, J. (2008). Teologia e Ciência. Diálogos acadêmicos em busca do saber. São Paulo: Educ/Paulinas.

-Tarnas, R. (2011). A Epopéia do Pensamento Ocidental. Para compreender as idéias que moldaram nossa visão de mundo. Rio de Janeiro: Bertrand Brasil.

-Teixeira, E., Müller, M. \& Da Silva, J. (2004). Espiritualidade e Qualidade de Vida. Porto Alegre: EDIPUCRS.

-Teixeira, F. (2014). O Resgate da espiritualidade no cotidiano. En R. Panasiewicz \& J. Vitório, Espiritualidades e Dinâmicas Sociais. Memória-Prospectivas (pp. 150-152). Belo Horizonte/São Paulo: Soter/Paulinas.

-Volcan, S., Sousa, P., Mari, J. \& Horta, B. (2003). Relação entre bem-estar espiritual e transtornos psiquiátricos menores: estudo transversal. Revista de Saúde Pública, 37(4), 440-445.

Sumario: Introducción; 1. Dificultades en la relación entre salud y espiritualidad; 2. Proyecciones en la relación entre salud y espiritualidad; 3. Relación entre salud y espiritualidad más allá de las dificultades y proyecciones; Consideraciones finales; Referencias. 